\title{
When Emotions are Triggered by Single Musical Notes: Revealing the Underlying Factors of Auditory-Emotion Associations
}

\author{
Patrick O'Toole \\ University College Cork \\ Cork, Cork, Ireland \\ patrick.otoole@umail.ucc.ie \\ Ian Pitt \\ University College Cork \\ Cork, Cork, Ireland \\ ianp@cs.ucc.ie
}

\author{
Donald Glowinski \\ University of Geneva \\ Geneva, Switzerland \\ donald.glowinski@unige.ch \\ Maurizio Mancini \\ Sapienza University of Rome \\ Rome, Italy \\ m.mancini@di.uniroma1.it
}

\begin{abstract}
Can emotion be experienced when the auditory sense is stimulated by a single musical note (Q1), and do variables such as musical skills, age, and personality traits have an influence in auditoryemotion associations (Q2)? An experiment was conducted, in which 130 participants were asked to listen to single musical notes and rate their experienced emotional state. They also had to rate their musical proficiency, sound sensitivity, strongest learning style, and complete a reduced version of the Big-Five personality test (BFI-10). Results regarding Q1 show a correlation between lower notes and sadness, and higher notes and joy, confirming previous auditoryemotion association research, while presenting new knowledge into how emotion associates with single musical notes. Results regarding Q2 show that musical proficiency (low vs high), learning style (aural vs physical), personality (level of Conscientiousness) had an effect on how participants emotionally experienced single musical notes. The results presented in this study will provide a starting point that can help develop a new auditory-visual framework that uses understandings on emotion, personality and other variables in the development of more personalised human-computer interfaces. This new framework can be used in applications that can help in learning to paint or play an instrument; promoting positive mental health, or exploring new forms of creative expression e.g., writing a song with a paint brush as the instrument or painting a picture with a piano as your brush.
\end{abstract}

\section{CCS CONCEPTS}

- Human-centered computing $\rightarrow$ Sound-based input / output; Interaction design process and methods; • Applied computing $\rightarrow$ Sound and music computing; • Social and professional topics $\rightarrow$ User characteristics.

This work is licensed under a Creative Commons Attribution International 4.0 License.

ICMI '21 Companion, October 18-22, 2021, Montréal, QC, Canada

(c) 2021 Copyright held by the owner/author(s).

ACM ISBN 978-1-4503-8471-1/21/10.

https://doi.org/10.1145/3461615.3485419

\section{KEYWORDS}

auditory-emotion associations, multi-modal interactions, music, personality

\section{ACM Reference Format:}

Patrick O’Toole, Donald Glowinski, Ian Pitt, and Maurizio Mancini. 2021. When Emotions are Triggered by Single Musical Notes: Revealing the Underlying Factors of Auditory-Emotion Associations. In Companion Publication of the 2021 International Conference on Multimodal Interaction (ICMI '21 Companion), October 18-22, 2021, Montréal, QC, Canada. ACM, New York, NY, USA, 8 pages. https://doi.org/10.1145/3461615.3485419

\section{INTRODUCTION}

This paper presents an experiment to help gain a deeper understanding into associations between single musical notes and emotions, and what impact different individual variables, such as personality, musical experience and learning style can have on auditory-emotion associations. Emotion and personality have become popular areas of research in recent years, especially in relation to technology $[12,33]$. With a great amount of personal data being captured by people's devices, companies are using this data to understand their users and personalise tools and products to each user. Multi-modal interaction is an area that can benefit from adopting more personalised approaches to how we form associations between our senses $[18,30]$. Interesting insights from a study, that trained participants in sound-colour associations using an ad-hoc program, while also investigating the association between sound and emotion of participants, were found. The fore-mentioned study found a correlation between very basic emotions (sadness and joy) and basic auditory stimuli (lower and higher pitch), but not at a significant level and with a low testing sample [31]. The motivation for our paper is to explore the understandings of associations between single musical notes and emotion, and if individual variables can play an important part in the process of auditory-emotion associations. With this new understanding, an improved model of auditory-visual associations can be applied in designing human-computer interfaces, one that takes account of emotion as well as personality, age, gender, and other variables. Figure 1 highlights the research of single musical notes-emotion associations, that is presented in this paper, as part of a larger framework of auditory-emotion-colour associations. This paper will help contribute to this larger framework 
of auditory-emotion-visual associations and contribute to future designs of intelligent and personalised human-computer interfaces, that can harness the power of multi-modal associations in creative and artistic digital environments.

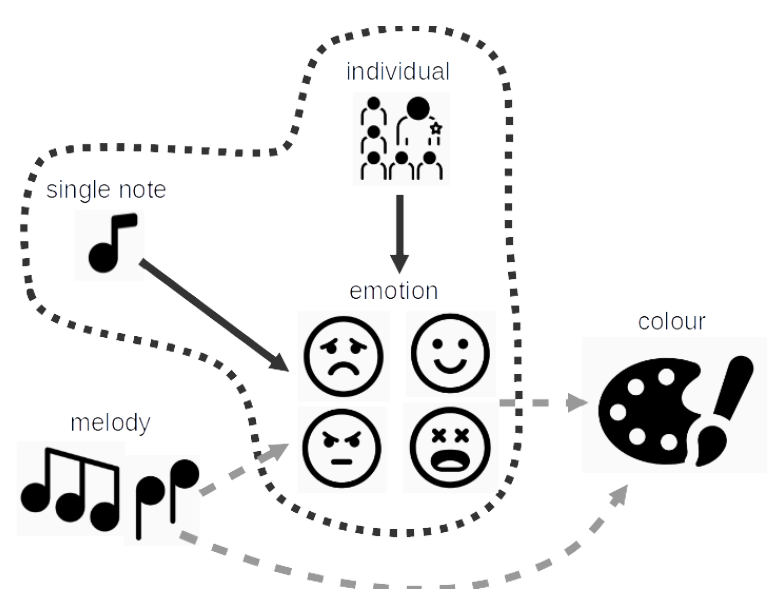

Figure 1: The work presented in the paper is highlighted by the dotted line: while cross-modal melody-emotion-colour and melody-colour associations are widely studied [20, 29, $32,36-40,42]$, we focus on single note-emotion associations by also looking at the individual variables in Section 2.2 , in the larger framework of studying sound-emotion-colour associations.

\section{BACKGROUND}

\subsection{Auditory-Emotion Research}

Previous research into associations between auditory stimuli and emotion has been mostly situated in auditory-visual cross-modal association research, that mainly explores the role of emotion in this relationship, mostly using musical excerpts instead of single musical notes $[4,6,21,28,37,46]$. Palmer et al. present the emotional meditation hypothesis, which is understood to be, when people listen to music, they have emotional responses and pick colors with similar emotional content [32]. The study from Palmer et al., and other studies have provided interesting insights into how auditory stimuli is perceived emotionally in relation to colors, with music in a major scale and at a faster tempo to be perceived lighter in colour and happier, with the opposite being true for a minor scale [32, $39,43]$. Other studies have focused solely on the auditory-emotion associations, however, these studies (like the ones mentioned above) focus on musical excerpts and not basic auditory stimuli [27, 40].

Very few studies have been conducted on the association between basic auditory stimuli, like single musical notes, and emotion $[2,26,37]$. Like Palmer et al., Spence also supports emotion mediation as one of the key factors in cross-modal correspondences, not just for complex auditory stimuli but also more simple stimuli [37]. Comparing simple auditory stimuli to more complex stimuli shows that emotion mediation counts for less variance in the empirical matching data, and therefore elicits less pronounced perceived emotion [37]. If we can understand auditory-emotion associations in a similar way to auditory-visual associations, that are mediated by emotion, lower notes (darker colours) should associate with negative emotions and higher notes (lighter colours) with positive emotions [29, 36].

Studies on the impact of different emotion models has been carried out in research around auditory-emotion associations, with three emotion models highlighted (Basic/discrete, dimensional and musically-induced) $[40,45]$. While the musically-induced emotion model would seem appropriate for a study on single musical noteemotion associations, the comparative test of these models and also information on personality bias help formulate the best approach for the experiment presented in this paper. The study by Zentner et al., showed that, compared to the other two models, the basic emotion model performed rather well when rating perceived emotions, even if the musically-induced model was more consistent [45]. In another study, the dimensional model performed better than both the basic and musically-induced emotion one in the discrimination of musical excerpts. However, "Personality-related differences were the most pronounced in the case of the discrete emotion model" [40]. Three different studies on emotion words expressed when listening to music stimuli, show that happiness, sadness, anger and fear were among the top choices across all three studies [23]. Taking into account the studies presented above, and bearing in mind that our study focuses on single musical notes rather than musical excerpts, and includes personality traits as an independent variable to be analysed, a basic emotion model (joy, sadness, anger and fear) is proposed.

\subsection{Individual Variables}

In this paper, we also seek to understand how twelve individual variables (Age, Gender, Musical Experience, Sound Sensitivity, Auditoryto-Other-Sense, Other-Sense-to-Auditory, Learning Style and the Big Five personality traits (BFI) of Openness, Conscientiousness, Extraversion, Agreeableness and Neuroticism), discussed in this section, can impact on the associations between single musical notes and emotion.

2.2.1 Personality traits. The relationship between personality traits and emotional responses to music is well researched. Studies have found significant correlations between the traits Neuroticism/Extraversion and positive/negative emotions [3, 8, 22]. Chamorro-Premuzic \& Furnham found that personality and intelligence can be a factor on how people use music. A person with a high IQ and high Extraversion might use music in a rational cognitive way, while someone with high Neuroticism and low Conscientiousness might use music for emotional regulation [8]. In another study, Neuroticism was found to significantly map to all negative emotions, while Extraversion significantly mapped to the positive emotions, "interest" and "enjoyment" as well as the negative emotion "shyness" [22]. From a review on present literature on the correlation between basic emotions and personality traits, limited findings regarding the traits of Openness to Experience, Conscientiousness and Agreeableness were found [15]. The Big Five personality test is a widely adopted test used in studies relating to personality traits. It is simple and easy to interpret, and is also an effective way to understand the personality traits of an individual. Rammstedt and John conducted research 
into the accuracy of the BFI-10 test (using only 10 questions) and found it sufficient to use in a research settings with limited time constraints, compared to the BFI-44 test (using 44 questions) [34].

2.2.2 Musical vs Non-Musical Experience. A study by Manno et al., investigated how musicians and non-musicians identify emotion in music. Music uses Temporal Fine Structures (TFS) and Envelope (ENV) modulation to resolve emotion in music, however the exact contributions of TFS and ENV is not known. The study showed that TFS is essential in identifying emotion in music and that there is a difference in how much TFS is used by musicians and nonmusicians. Non-musicians use less fine structure information and have reduced emotional resolvability curves compared to musicians [27]. While the study above shows that musical experience can have a difference in how much TFS is used in identifying emotion in music, it is unclear if the same logic can be adopted when identifying emotion from single musical notes. A study on expressive intentions with single piano notes, shows that musical expertise has no mean effect on performers when tested on the four acoustical parameters of pitch, intensity, articulation and rhythmic density, with both musicians and non-musicians using these acoustic parameters in very similar ways when listening to a single piano note [2].

2.2.3 Age and Gender. Studies show that age can have an impact on how we associate between our senses and how we use emotion in response to stimuli, and that it can change over time [21, 35, 44]. Hunter et al., using two variants of an emotional Stroop task, found that older adults can match congruent cross-modal stimuli just as well as younger adults however, with incongruent stimuli, the older adults performed worse than their younger counterparts [21]. Older adults tend to weaken the ability to perceive negative emotions the older they get [35], this can be caused by the fact they tend to remove problematic relationships and avoid interpersonal conflict, leading to a more positive social environment $[1,5]$.

As well as age, studies into the role of gender in auditory-emotion associations research has shown some interesting insights. Studies between men and women with regards to both uni and multi sensory emotional stimuli have shown that women are more accurate than men in recognition of emotional prosody [10, 24, 41].

2.2.4 Other Variables. Sound sensitivity, in general and when sound is triggered as the primary and secondary source, are also investigated in this paper. Misophonia is a condition characterized by heightened emotional reactivity to common repetitive sounds, accompanied by difficulties responding to these sounds and associated impairment in functioning [7]. A study on the relationship between the Neuroticism trait and Misophonia, with regard to the role of emotion regulation found that "difficulties with emotion regulation and Neuroticism were significantly positively correlated with symptoms of misophonia" [7]. Past research in auditory-visual cross-modal associations and chromaesthesia have also informed our understanding of the way in which perception operates between auditory/other sense and other sense/auditory with regard to sound sensitivity [11, 26, 36].

Seven learning-style terms from Gardner's Theory of Multiple Intelligences (Aural, Visual, Logical, Verbal, Physical, Social Interpersonal and Solitary Intrapersonal) have been used to understand the different ways people learn and take in information [16]. While the method used in our study to understand learning styles relies on the participants perception of the strongest learning style, and not assessed with a well-known method, like the BFI test for personality traits, some interesting insights that can inform future work is expected.

\section{EXPERIMENT}

This experiment looks to understand associations between single musical notes and basic emotions, and how other independent variables impact these associations, with two specific questions in mind:

- Q1 - Can emotion be experienced from a single musical note and if so, how basic a level is the emotional perception that is experienced?

- Q2 - Is there a significant correlation between auditoryemotion choices when factoring in the twelve independent variables mentioned in Section 2.2?

\subsection{Participants}

130 people $($ female $=95$, male $=34$, other $=1$ ) participated in the online survey, with participants identifying with the following age groups: 35-44 (38), 24-34 (32), 45-55 (29), 55-64 (14), 18-24 (9), over 65 (6) and under 18 (2). Information was also gathered on participants' musical experience ( $82 \%$ with musical experience and $18 \%$ with no experience) and creativeness (61\%, regularly partaking in a creative hobby; $24 \%$, not so regularly; and $15 \%$, with no creative hobby). While musical experience was used as one of the individual variables that could impact the emotion response to auditory stimuli, the questions regarding musical instruments played by participants and their level of creative expression was used to gather an extra layer of understanding to the results of the experiment.

\subsection{Sound and Emotion Stimuli}

In this experiment, the auditory stimuli were recorded on the Logic Pro X software using a Fender Telecaster electric guitar as the instrument. Each note was recorded on a mono track with each note lasting 6 seconds with a smooth short fade at the end of each clip. A basic EQ, compressor and room reverb are used to obtain a more natural sound, with each sound file rendered to an mp3 file with the normalised function turned on. The envelope of the recorded notes consist of a sharp attack to the maximum amplitude, with a steady decay as the note rings out. As this was an online survey, we had no control of the absolute volume participants listened to the stimuli, but asked them to set it to a comfortable listening level. The first octave of the guitar was chosen to elicit the auditory stimuli, from note E2 $(82.41 \mathrm{~Hz})$ to note D\#3 $(155.6 \mathrm{~Hz})$. Final Cut Pro was used to create short video files with a white background with black text counting down from three, with audio stimuli playing on zero. These video files provided better functionality in Limesurvey ${ }^{1}$, than audio only files and also added a visual instruction to participants. For the emotion stimuli, the four emotion words, sadness, anger, fear and joy were used. These emotions are taken from the discrete emotion model mentioned in Section 2.1.

\footnotetext{
${ }^{1}$ https://www.limesurvey.org
} 


\subsection{Procedure}

The online survey was distributed through the University College Cork survey mailing list, as well as being shared on LinkedIn and Twitter. Once participants clicked on the survey link, they landed on the welcome page and were presented with a detailed explanation of the survey, as well as contact information of the researchers, if they had any questions regarding the survey and could click "Next" to agree to start the survey. The survey was broken up into four sections: General Information, Personality Test, Crossmodal Association Questions and Auditory-emotion Questions. The survey is described in following four sections.

3.3.1 General Questions Section. Each section was displayed on a separate page with participants instructed to click the "Next" button to move to the next section. The first section contained five questions regarding age, gender, musical ability, instruments played (this question only appeared if the participant had stated they had musical experience in the previous question) and creative hobbies.

3.3.2 Personality Test Section. This section involved the participants taking the short Big Five personality test (BFI-10). Participants responded to ten questions on a five point Likert scale ranging from "Strongly Disagree" to "Strongly Agree". These questions were randomised to avoid any bias in the survey. After the ten questions were answered, participants could consent to share their email address to get their BFI-10 scores sent to them at a later time. If consent was given, the participant could input their email address on the next page.

3.3.3 Cross-modal Association Section. The third section presented four questions, with the first three designed to gain an understanding of the participants' relationship with sound, and how other senses impact and are impacted by auditory stimuli. The fourth question looked to find what learning style participants associated with their style of learning. The participants rated the first three questions, between 1 (weak) and 5 (strong) in sensitivity/sensation. The first question asked participants to rate their sensitivity to noise/sound; the next two questions asked participants to rate the sensation strength in other senses when the auditory sense is the primary trigger (i.e., when a bell is rung and triggers sensation), and in the auditory sense when the other sense is the primary trigger (i.e., when a flash of light triggers sensation). For the last question participants were given the options of Visual, Logical, Aural, Verbal, Physical, Social Interpersonal and Solitary Interpersonal learning styles from Section 2.2.4, and asked to choose their top three, in order of perceived strongest to weakest.

3.3.4 Auditory-emotion Questions Section. The final section contained twelve questions presented in randomised order, to avoid selection bias. Each question contained a nine second video, which instructed participants to press play when ready. The video presented a count down from three with the auditory stimuli playing on zero, giving the participant time to fully listen to the note played. As mentioned in Section 3.2, the first octave of the guitar was used for the auditory stimuli. They were informed to listen once and to choose an emotion word from the options presented (Sadness, Joy, Anger, Fear).
Percentage of emotion choice by musical notes

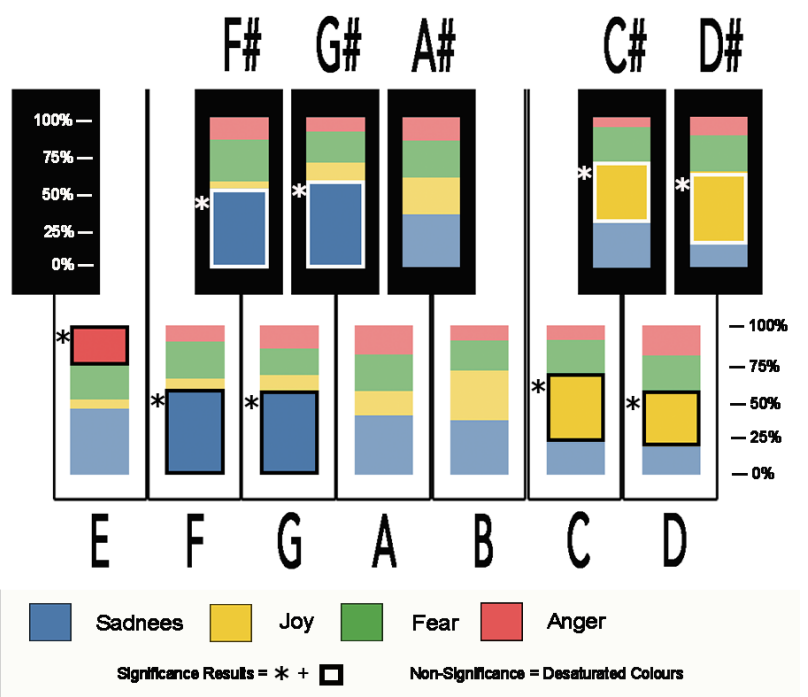

Figure 2: This figure shows the percentage between the four emotion words that participants chose for each musical note. Sadness significantly ( ${ }^{*}$ ) associated with the lower notes, with Joy significantly $\left(^{*}\right)$ associating with the higher notes.

Once the twelve sound-emotion questions were answered, they were directed to submit the survey and a conclusion page was displayed thanking participants for taking part.

\subsection{Data Handling and Analysis}

The survey data was stored within the Limesurvey application running from the researcher's hosting site. All data was anonymised and information on IP addresses, date stamp and referrer URLs were not collected. Email addresses were collected only from the participants who consented to leave it for the purpose of sharing the results of the personality test. The email data was deleted once the results were obtained and emailed to the participants.

To answer Q1, a chi-square test was conducted on the cross-table Note by Emotion. The chi-square test was significant $\left(\left(\chi^{2}(33 \mathrm{n}=\right.\right.$ $1560)=274.39, \mathrm{p}<.001)$, with a small effect size (Cramer's V =.24).

To answer Q2, a multinomial stepwise multiple regression model was carried out in which the dependent variable was the single musical notes. Using the variables obtained from the data in the survey as models, Age, Gender, Musical Experience, Sound Sensitivity, Auditory-to-Other-Sense, Other-Sense-to-Auditory, Learning Style and the five personality traits of Openness, Conscientiousness, Extra-version, Agreeableness and Neuroticism were analysed.

\subsection{Results}

3.5.1 Q1 - Auditory-emotion association. For Q1, the results in Table 1 show that very basic emotion can be experienced from basic auditory stimuli, like a single musical note. For four out of five of the lowest notes (E,F,F\#,G,G\#), Table 1 shows that the adjusted standardised residual (ASR) scores are significantly higher for Sadness. The one exception was the lowest note (E) where Anger has a 
Table 1: Results of the chi-square test that was conducted on the cross-table Note-by-Emotion. The chi-square test was significant $\left(\chi^{2}(33 \mathrm{n}=1560)=274.39, \mathrm{p}<.001\right)$, with a small effect size (Cramer's $V=.24)$. The significant ARSs are highlighted in bold.

\begin{tabular}{crrrrr}
\hline & & \multicolumn{4}{c}{ Emotion } \\
\hline Note & & Sadness & Anger & Fear & \multicolumn{1}{c}{ Joy } \\
D\# & Count & $21 \mathrm{a}$ & $16 \mathrm{a}, \mathrm{b}$ & $31 \mathrm{~b}$ & $62 \mathrm{c}$ \\
& Adj. Res. & $\mathbf{- 5 . 5 8 6}$ & -0.593 & 0.217 & $\mathbf{6 . 6 6 4}$ \\
D & Count & $25 \mathrm{a}$ & $26 \mathrm{~b}$ & $31 \mathrm{~b}$ & $48 \mathrm{~b}$ \\
& Adj. Res. & $\mathbf{- 4 . 8 3 5}$ & 2.044 & 0.217 & $\mathbf{3 . 6 5 4}$ \\
C\# & Count & $39 \mathrm{a}$ & $8 \mathrm{a}$ & $30 \mathrm{a}, \mathrm{b}$ & $53 \mathrm{~b}$ \\
& Adj. Res. & -2.206 & -2.703 & 0.000 & $\mathbf{4 . 7 2 9}$ \\
C & Count & $29 \mathrm{a}$ & $13 \mathrm{a}$ & $29 \mathrm{a}$ & $59 \mathrm{~b}$ \\
& Adj. Res. & $\mathbf{- 4 . 0 8 4}$ & -1.384 & -0.217 & $\mathbf{6 . 0 1 9}$ \\
B & Count & $48 \mathrm{a}$ & $13 \mathrm{a}$ & $26 \mathrm{a}$ & $43 \mathrm{a}$ \\
& Adj. Res. & -0.516 & -1.384 & -0.870 & 2.580 \\
A\# & Count & $46 \mathrm{a}$ & $20 \mathrm{a}$ & $32 \mathrm{a}$ & $32 \mathrm{a}$ \\
& Adj. Res. & -0.892 & 0.461 & 0.435 & 0.215 \\
A & Count & $52 \mathrm{a}$ & $25 \mathrm{a}$ & $32 \mathrm{a}$ & $21 \mathrm{a}$ \\
& Adj. Res. & 0.235 & 1.780 & 0.435 & -2.150 \\
G\# & Count & $75 \mathrm{a}$ & $12 \mathrm{~b}$ & $27 \mathrm{a}, \mathrm{b}$ & $16 \mathrm{~b}$ \\
& Adj. Res. & 4.554 & -1.648 & -0.652 & -3.224 \\
G & Count & $73 \mathrm{a}$ & $20 \mathrm{a}, \mathrm{b}$ & $23 \mathrm{~b}, \mathrm{c}$ & $14 \mathrm{c}$ \\
& Adj. Res. & $\mathbf{4 . 1 7 8}$ & 0.461 & -1.522 & $\mathbf{- 3 . 6 5 4}$ \\
F\# & Count & $69 \mathrm{a}$ & $19 \mathrm{a}$ & $36 \mathrm{a}$ & $6 \mathrm{~b}$ \\
& Adj. Res. & $\mathbf{3 . 4 2 7}$ & 0.198 & 1.305 & $\mathbf{- 5 . 3 7 4}$ \\
F & Count & $74 \mathrm{a}$ & $14 \mathrm{a}, \mathrm{b}$ & $32 \mathrm{a}$ & $10 \mathrm{~b}$ \\
& Adj. Res. & $\mathbf{4 . 3 6 6}$ & -1.121 & 0.435 & $\mathbf{- 4 . 5 1 4}$ \\
E & Count & $58 \mathrm{a}$ & $33 \mathrm{a}$ & $31 \mathrm{a}$ & $8 \mathrm{~b}$ \\
& Adj. Res. & 1.361 & 3.890 & 0.217 & $\mathbf{- 4 . 9 4 4}$
\end{tabular}

higher ASR score but not at a significant level; however, Sadness was chosen by most participants, but not at a significant level. For the higher notes, results show that the four highest notes (C,C\#,D,D\#) are significantly higher for foy in the ASR scores. Table 1 also highlights that only the emotions of foy and Sadness found significance, either positively or negatively in ASR scores, that could suggest very basic emotion associations to single musical notes. Results show that for notes $A, A \#$, and $B$, which are placed in the middle of the octave of notes tested, no significant statistics are shown.

3.5.2 Q2 - Impact of Independent Variables on Sound-emotion Association. Results pertaining to Q2 highlighted some interesting insights. Q2 looked at how the individual variables, mentioned in Section 2.2, can affect the emotion choice for each single musical note. Analysis was performed on each note, and Table 2 shows significant results at $p<.05$ where the individual variables had an impact on the association between single musical notes and emotion. As shown in Table 2, six out of the twelve musical notes showed statistical significance in relation to one or more predictors. The odds ratio column in Table 2 represents the relative likelihood of each predictor type yielding a choice for a particular emotion. For example, Table 2 shows that a person with a higher sensitivity to sound is 2.308 times more likely to choose Anger than foy (the emotion used as a reference) in relation to the $E$ note. For individual variables with an odds ratio of less than 1 , the predictor is less likely to choose the stated emotion than the referenced emotion foy, i.e., participants with musical proficiency are less likely to experience Sadness, Anger and Fear compared to foy.

\subsection{Discussion}

3.6.1 Q1 - Sound-emotion Association. Similar to the findings of O'Toole et al., associations between basic auditory stimuli and emotion can be viewed at a very basic level of emotional perception [31]. The results from the chi square test, illustrated in Table 1, show a significant association between Lower-notes/Sadness and Highernotes/Joy. In Section 2.1, research on different emotion models were presented, with the basic/discrete model considered the most appropriate for an experiment using single musical notes and for perceiving rather than feeling emotions [45]. For future research, adopting a multi-modal approach that can understand multiple features from auditory stimuli would be beneficial. However for this study on basic auditory stimuli, results show a basic emotion choice for single musical notes, validating the use of the basic emotion model in this experiment.

With the three notes $(A, A \#, B)$ in the middle of the octave that showed no significance, all three notes had Sadness as the most chosen emotion. A Study by Chau et al. has shown that the timbre of an instrument can impact the emotion response when hearing a sound. Results showed that the guitar rated higher for negative emotional characteristics compared to a piano (which was emotionally neutral) and instruments like a vibraphone or marimba (which was emotionally positive) [9]. Findings from another study found that "timbre independently affects the perception of emotions in music after controlling for other acoustic, cognitive and performance factors" [19] While timbre is one of many acoustic properties that can impact the emotion response to sound, if we are analysing single musical notes that lack tempo, rhythm and modes, it could have a bigger impact on how a person responds to sound emotionally. To answer Q1, emotion can be experienced from basic auditory stimuli, such as a single musical note at a very basic level of emotion perception. Without melody, harmony, rhythm, modes and other audio features a single note can be perceived to be mostly happy or sad and this is mostly dependant on pitch.

3.6.2 Q2 - Independent Variables Impact on Sound-emotion Association. Q2 investigated how different individual variables can impact a participants' emotion word choices. In Table 2, significant scores in seven out of twelve individual variables across six of the twelve musical notes were found.

Conscientiousness provided a significant negative correlation with Anger on the note $A$. This result would fit the personality trait. Previous studies have shown that the Conscientiousness trait is positively associated with the optimistic-conventional dimension in relation to musical preference [14]. Studies have shown that a person high in Conscientiousness is likely to; like high tempo music in a major key rather than low tempo in the minor key [14]; use music in a more cognitive rather than an emotional way [8]; and usually can regulate or control impulses better [13].

Table 2 shows that musically proficient participants significantly chose foy over Sadness, Anger and Fear when listening to the note A. 
Table 2: Result of multinomial stepwise multiple regression model. Only the significant predictors are reported. The reference emotion is always Joy.

\begin{tabular}{|c|c|c|c|c|c|c|c|c|}
\hline & & \multirow[t]{2}{*}{ Estimate } & \multirow{2}{*}{$\begin{array}{l}\text { Std. } \\
\text { Error }\end{array}$} & \multirow[t]{2}{*}{ Wald } & \multirow[t]{2}{*}{ Sig. } & \multirow{2}{*}{$\begin{array}{l}\text { Odds } \\
\text { ratio }\end{array}$} & \multicolumn{2}{|c|}{ 95\% Conf. Int. } \\
\hline & & & & & & & L Bound & U Bound \\
\hline \multicolumn{9}{|c|}{ Note: C\# } \\
\hline Anger & Male & 1.946 & 0.811 & 5.762 & .016 & 7.000 & 1.429 & 34.286 \\
\hline Fear & Male & 1.302 & 0.508 & 6.573 & .010 & 3.675 & 1.359 & 9.940 \\
\hline \multicolumn{9}{|c|}{ Note: C } \\
\hline Anger & Aural & 1.946 & 0.886 & 4.818 & .028 & 7.001 & 1.232 & 39.766 \\
\hline Anger & Physical & 1.946 & 0.976 & 3.976 & .046 & 7.001 & 1.034 & 47.418 \\
\hline \multicolumn{9}{|c|}{ Note: A } \\
\hline Sadness & Proficiency & -1.034 & 0.324 & 10.186 & .001 & .355 & .188 & .671 \\
\hline Anger & Proficiency & -0.825 & 0.365 & 5.102 & .024 & .438 & .214 & .897 \\
\hline Anger & Conscientiousness & -0.051 & 0.024 & 4.550 & .033 & .950 & .906 & .996 \\
\hline Fear & Proficiency & -1.042 & 0.353 & 8.728 & .003 & .353 & .177 & .704 \\
\hline \multicolumn{9}{|c|}{ Note: G\# } \\
\hline Anger & age & -0.817 & 0.360 & 5.146 & .023 & .442 & .218 & .895 \\
\hline Fear & age & -0.853 & 0.307 & 7.746 & .005 & .426 & .234 & .777 \\
\hline \multicolumn{9}{|c|}{ Note: G } \\
\hline Anger & age & -1.095 & 0.345 & 10.084 & .001 & .335 & .170 & .658 \\
\hline \multicolumn{9}{|c|}{ Note: E } \\
\hline Anger & sensitive_sound & 0.836 & 0.373 & 5.022 & .025 & 2.308 & 1.111 & 4.795 \\
\hline
\end{tabular}

This correlation could be explained by the fact that the $A$ note is the standard tuning note on the guitar. 34 participants were musically proficient, with guitar as their choice instrument. Hearing this particular note could have contributed to elicit positive emotions, and it could be possible that other guitar players have an overall more positive emotion perception when hearing sounds from a guitar.

It could be also similar to both the optimistic (foy) and the conventional (standard tuning note) dimensions for the Conscientiousness trait mentioned above. While both musicians and nonmusicians can have an understanding of communicating expressive content, as presented in Section 2.2.2, musicians use a greater amount of temporal fine structure (TFS) information and a higher emotional resolvability curve [27]. This could impact how musicians respond to musical notes in different ways to non-musicians and why musical proficiency correlates to foy with regard to the $A$ note.

Table 2 shows interesting correlations between the Note $C$ and the Aural and Physical learning styles. Participants who chose Aural or Physical as their preferred learning style were around seven times more likely to chose Anger. Studies have shown that, when learning an instrument, aural learning was the main learning style, but participants also used more logical and practical approaches when learning new songs [17]. For aural learners, with musical experience (87.5\% of aural learners), this correlation between the $C$ note and the Aural learning style could be due to frustration of not having the option to use more logical and practical learning styles to help them understand the musical note being elicited. Looking at the positive correlation between Anger and the Physical learning style for the note $C$, some interesting insights can be seen. The $C$ note is the fourth highest frequency tested in this study and while overall we can see from Table 1 that foy is the significant emotion, higher frequencies can also garner annoyance in some people. A study measuring noise annoyance levels in working environments has shown that higher frequencies had the highest annoyance rating with the lowest frequencies having the lowest annoyance rating [25]. It is possible that the Anger associated with the Physical, as well as the Aural learning styles, comes from the higher frequency of the $C$ note.

\section{CONCLUSIONS}

In this paper, we presented an experiment on auditory-emotion associations, looking to reveal the emotional potential of an apparentlybasic stimulus such as a single musical note and the impact of other variables such as, personality, gender, age and musical experience on this association. Through this approach, we were able to more clearly distinguish the combination of key factors accounting for associations between auditory stimuli and emotion. Results showed significance on basic associations in lower notes to sadness and higher notes to joy, with a positive correlation between the four highest notes and the emotion joy, and a positive correlation between three of the four lowest notes and the emotion, sadness. Results pertaining to the impact of individual variables on auditory-emotion associations showed significant results on six of the twelve musical notes. The independent variables of gender, 
aural and physical learning styles, musical proficiency, the Conscientiousness personality trait, age and sound sensitivity were found to be significant variables in six of the twelve single musical notes, thus potentially impacting the emotional response to the auditory stimuli.

Some limitations of this experiment include, for example, the use of only one octave and the choice of instrument for auditory stimuli. One octave was used in this experiment, to reproduce the test setup from [31], with a larger test group. In the future, it would be beneficial to study auditory-emotion associations in a range of octaves, to understand if pitch height-emotion associations are the most dominant associations regarding single musical notes. Also, the use of a single instrument type is an important factor in associations between single musical notes and emotions. Each instrument has a different timbre which elicits different emotion characteristics. As mentioned in Section 3.6.1, it has been shown that the timbre of the guitar is highly rated for negative emotional characteristics, where a Piano is rated as neutral, and a vibraphone is highly rated for positive emotional characteristics [9]. Changing the instrument that elicits auditory stimuli, by adopting a piano (neutral emotional characteristics) or using multiple instrument sounds, with different emotional characteristics is important for future studies and will help understand single musical note-emotion associations and the impact of timbre and multiple octaves have on the emotion response.

The findings of the experiment presented in the paper can help towards the formulation of a new auditory-visual framework that uses understandings on emotion, personality and other variables in the development of more personalised human-computer interfaces. These findings can improve the design of programs such as SoundStrokes presented in [31], that can assist in sharpening our associations between our senses. Further research into the aforementioned auditory-visual framework could lead to the creation of applications using computer-generated cross-modal stimuli, e.g., synthesised audio-visual tutorials, to learn or improve skills. These applications could be used for a multitude of purposes, such as learning an instrument or to paint; and in exploring new creative ways of expression, e.g., writing a song with a paint brush as the instrument or painting a picture with a piano as your bush. Creativity should not be limited to one form of expression and understanding the intricacies of our senses, combined with emotion, personality traits and other variables, it can provided a platform for multi-modal expression using digital tools.

\section{ACKNOWLEDGMENTS}

This publication has emanated from research supported in part by a Grant from Science Foundation Ireland under Grant number $18 / \mathrm{CRT} / 6222$.

\section{REFERENCES}

[1] Toni Antonucci, Hiroko Akiyama, and Keiko Takahashi. 2004. Attachment and Close Relationships across the Life Span. Attachment \& Human Development 6, 4 (Dec. 2004), 353-370. https://doi.org/10.1080/1461673042000303136

[2] Filippo Bonini Baraldi, Giovanni De Poli, and Antonio Rodà. 2006. Communicating Expressive Intentions with a Single Piano Note. fournal of New Music Research 35, 3 (Sept. 2006), 197-210. https://doi.org/10.1080/09298210601045575

[3] Lisa Feldman Barrett. 1998. Discrete Emotions or Dimensions? The Role of Valence Focus and Arousal Focus. Cognition \& Emotion 12, 4 (July 1998), 579-599. https://doi.org/10.1080/026999398379574
[4] Thomas Baumgartner, Michaela Esslen, and Lutz Jäncke. 2006. From Emotion Perception to Emotion Experience: Emotions Evoked by Pictures and Classical Music. International fournal of Psychophysiology 60, 1 (April 2006), 34-43. https: //doi.org/10.1016/j.ijpsycho.2005.04.007

[5] Kira S. Birditt and Karen L. Fingerman. 2005. Do We Get Better at Picking Our Battles? Age Group Differences in Descriptions of Behavioral Reactions to Interpersonal Tensions. The fournals of Gerontology Series B: Psychological Sciences and Social Sciences 60, 3 (May 2005), P121-P128. https://doi.org/10.1093/ geronb/60.3.P121

[6] Roberto Bresin. 2004. Real-Time Visualization of Musical Expression.. In $H U$ MAINE Workshop "From Signals to Signs of Emotion and Vice Versa". Santorini, 5.

[7] Clair Cassiello-Robbins, Deepika Anand, Kibby McMahon, Rachel Guetta, Jacqueline Trumbull, Lisalynn Kelley, and M. Zachary Rosenthal. 2020. The Mediating Role of Emotion Regulation Within the Relationship Between Neuroticism and Misophonia: A Preliminary Investigation. Frontiers in Psychiatry 11 (Aug. 2020), 847. https://doi.org/10.3389/fpsyt.2020.00847

[8] Tomas Chamorro-Premuzic and Adrian Furnham. 2007. Personality and Music: Can Traits Explain How People Use Music in Everyday Life? British fournal of Psychology 98, 2 (2007), 175-185. https://doi.org/10.1348/000712606X111177

[9] Chuck-Jee Chau, Bin Wu, and Andrew Horner. 2015. The Emotional Characteristics and Timbre of Nonsustaining Instrument Sounds. Fournal of the Audio Engineering Society 63, 4 (April 2015), 228-244. https://doi.org/10.17743/jaes. 2015.0016

[10] Oliver Collignon, Simon Girard, Frédéric Gosselin, Dave Saint-Amour, Franco Lepore, and Maryse Lassonde. 2010. Women Process Multisensory Emotion Expressions More Efficiently than Men. Neuropsychologia 48, 1 (Jan. 2010), 220225. https://doi.org/10.1016/j.neuropsychologia.2009.09.007

[11] Caroline Curwen. 2018. Music-Colour Synaesthesia: Concept, Context and Qualia. Consciousness and Cognition 61 (May 2018), 94-106. https://doi.org/10.1016/j. concog.2018.04.005

[12] Richard J Davidson, Klaus R Scherer, and H. Hill Goldsmith. 2009. Handbook of Affective Sciences. Oxford University Press, New York; Oxford.

[13] Colin G. DeYoung. 2010. Personality Neuroscience and the Biology of Traits: Personality Neuroscience. Social and Personality Psychology Compass 4, 12 (Dec. 2010), 1165-1180. https://doi.org/10.1111/j.1751-9004.2010.00327.x

[14] Snježana Dobrota and Ina Reić Ercegovac. 2015. The Relationship between Music Preferences of Different Mode and Tempo and Personality Traits - Implications for Music Pedagogy. Music Education Research 17, 2 (April 2015), 234-247. https: //doi.org/10.1080/14613808.2014.933790

[15] Ryan Donovan, Aoife Johnson, Aine deRoiste, and Ruairi O’Reilly. 2020. Quantifying the Links Between Personality Sub-Traits and the Basic Emotions. In Computational Science and Its Applications - ICCSA 2020, Osvaldo Gervasi, Beniamino Murgante, Sanjay Misra, Chiara Garau, Ivan Blečić, David Taniar, Bernady O. Apduhan, Ana Maria A.C. Rocha, Eufemia Tarantino, Carmelo Maria Torre, and Yeliz Karaca (Eds.). Vol. 12250. Springer International Publishing, Cham, 521-537. https://doi.org/10.1007/978-3-030-58802-137

[16] Howard Gardner. 1993. Frames of Mind: The Theory of Multiple Intelligences (2nd ed ed.). Fontana Press, London.

[17] Lucy Green. 2012. Musical "Learning Styles" and "Learning Strategies" in the Instrumental Lesson: Some Emergent Findings from a Pilot Study. Psychology of Music 40, 1 (Jan. 2012), 42-65. https://doi.org/10.1177/0305735610385510

[18] Annaliese Micallef Grimaud, Tuomas Eerola, and Nick Collins. 2019. EmoteControl: A System for Live-Manipulation of Emotional Cues in Music. In Proceedings of the 14th International Audio Mostly Conference: A fourney in Sound. ACM, Nottingham United Kingdom, 111-115. https://doi.org/10.1145/3356590.3356608

[19] Julia C. Hailstone, Rohani Omar, Susie M. D. Henley, Chris Frost, Michael G. Kenward, and Jason D. Warren. 2009. It's Not What You Play, It's How You Play It: Timbre Affects Perception of Emotion in Music. Quarterly fournal of Experimental Psychology 62, 11 (Nov. 2009), 2141-2155. https://doi.org/10.1080/ 17470210902765957

[20] Kate Hevner. 1936. Experimental Studies of the Elements of Expression in Music. The American fournal of Psychology 48, 2 (April 1936), 246. https: //doi.org/10.2307/1415746

[21] Edyta Monika Hunter, Louise H. Phillips, and Sarah E. MacPherson. 2010. Effects of Age on Cross-Modal Emotion Perception. Psychology and Aging 25, 4 (Dec. 2010), 779-787. https://doi.org/10.1037/a0020528

[22] Carroll E. Izard, Deborah Z. Libero, Priscilla Putnam, and O. Maurice Haynes. 1993. Stability of Emotion Experiences and Their Relations to Traits of Personality. Journal of Personality and Social Psychology 64, 5 (1993), 847-860. https://doi. org/10.1037/0022-3514.64.5.847

[23] Patrik N. Juslin. 2013. What Does Music Express? Basic Emotions and Beyond. Frontiers in Psychology 4 (2013). https://doi.org/10.3389/fpsyg.2013.00596

[24] Lena Lambrecht, Benjamin Kreifelts, and Dirk Wildgruber. 2014. Gender Differences in Emotion Recognition: Impact of Sensory Modality and Emotional Category. Cognition and Emotion 28, 3 (April 2014), 452-469. https: //doi.org/10.1080/02699931.2013.837378 
[25] Ulf Landström, Elisabeth Åkerlund, Anders Kjellberg, and Maria Tesarz. 1995 Exposure Levels, Tonal Components, and Noise Annoyance in Working Environments. Environment International 21, 3 (Jan. 1995), 265-275. https: //doi.org/10.1016/0160-4120(95)00017-F

[26] Lawrence E Marks. 2004. Cross-Modal Interactions in Speeded Classification. In The Handbook of Multisensory Processes, Gemma Calvert, Charles Spence, and Barry E. Stein (Eds.). MIT Press, Cambridge, Mass, 85-105.

[27] Francis A. M. Manno, Raul R. Cruces, Condon Lau, and Fernando A. Barrios. 2019. Uncertain Emotion Discrimination Differences Between Musicians and Non-Musicians Is Determined by Fine Structure Association: Hilbert Transform Psychophysics. Frontiers in Neuroscience 13 (Sept. 2019). https://doi.org/10.3389/ fnins.2019.00902

[28] Solange Mardaga and Michel Hansenne. 2009. Do Personality Traits Modulate the Effect of Emotional Visual Stimuli on Auditory Information Processing? Journal of Individual Differences 30, 1 (Jan. 2009), 28-34. https://doi.org/10.1027/16140001.30.1.28

[29] Gail Martino and Lawrence E Marks. 2000. Cross-Modal Interaction between Vision and Touch: The Role of Synesthetic Correspondence. Perception 29, 6 (June 2000), 745-754. https://doi.org/10.1068/p2984

[30] Vincenzo Moscato, Antonio Picariello, and Giancarlo Sperli. 2020. An Emotional Recommender System for Music. IEEE Intelligent Systems (2020), 1-1. https //doi.org/10.1109/MIS.2020.3026000

[31] Patrick O’Toole, Donald Glowinski, and Maurizio Mancini. 2019. Understanding Chromaesthesia by Strengthening Auditory-Visual-Emotional Associations. In 2019 8th International Conference on Affective Computing and Intelligent Interaction (ACII). IEEE, Cambridge, United Kingdom, 1-7. https://doi.org/10.1109/ACII. 2019.8925465

[32] Stephen E. Palmer, Karen B. Schloss, Zoe Xu, and Lilia R. Prado-Leon. 2013 Music-Color Associations Are Mediated by Emotion. Proceedings of the National Academy of Sciences 110, 22 (May 2013), 8836-8841. https://doi.org/10.1073/pnas. 1212562110

[33] Rosalind W Picard. 1997. Affective Computing. MIT Press, Cambridge, Mass.

[34] Beatrice Rammstedt and Oliver P. John. 2007. Measuring Personality in One Minute or Less: A 10-Item Short Version of the Big Five Inventory in English and German. Journal of Research in Personality 41, 1 (Feb. 2007), 203-212. https: //doi.org/10.1016/j.jp. 2006.02.001

[35] Ted Ruffman, Julie D. Henry, Vicki Livingstone, and Louise H. Phillips. 2008 A Meta-Analytic Review of Emotion Recognition and Aging: Implications for Neuropsychological Models of Aging. Neuroscience \& Biobehavioral Reviews 32
4 (Jan. 2008), 863-881. https://doi.org/10.1016/j.neubiorev.2008.01.001

[36] Charles Spence. 2011. Crossmodal Correspondences: A Tutorial Review. Attention, Perception, \& Psychophysics 73, 4 (May 2011), 971-995. https://doi.org/10.3758/ s13414-010-0073-7

[37] Charles Spence. 2020. Assessing the Role of Emotional Mediation in Explaining Crossmodal Correspondences Involving Musical Stimuli. Multisensory Research 33, 1 (July 2020), 1-29. https://doi.org/10.1163/22134808-20191469

[38] Xiuwen Sun, Xiaoling Li, Lingyu Ji, Feng Han, Huifen Wang, Yang Liu, Yao Chen, Zhiyuan Lou, and Zhuoyun Li. 2018. An Extended Research of Crossmodal Correspondence between Color and Sound in Psychology and Cognitive Ergonomics. Peerf 6 (March 2018), e4443. https://doi.org/10.7717/peerj.4443

[39] Tawney Tsang and Karen B. Schloss. 2010. Associations between Color and Music Are Mediated by Emotion and Influenced by Tempo: (525772013-006). https://doi.org/10.1037/e525772013-006

[40] Jonna K. Vuoskoski and Tuomas Eerola. 2011. Measuring Music-Induced Emotion: A Comparison of Emotion Models, Personality Biases, and Intensity of Experiences. Musicae Scientiae 15, 2 (July 2011), 159-173. https://doi.org/10. $1177 / 1029864911403367$

[41] Teija Waaramaa. 2017. Gender Differences in Identifying Emotions from Auditory and Visual Stimuli. Logopedics Phoniatrics Vocology 42, 4 (Oct. 2017), 160-166. https://doi.org/10.1080/14015439.2016.1243725

[42] J Ward, B Huckstep, and E Tsakanikos. 2006. Sound-Colour Synaesthesia: To What Extent Does It Use Cross-Modal Mechanisms Common to Us All? Cortex 42, 2 (2006), 264-280. https://doi.org/10.1016/S0010-9452(08)70352-6

[43] Kelly L. Whiteford, Karen B. Schloss, Nathaniel E. Helwig, and Stephen E. Palmer. 2018. Color, Music, and Emotion: Bach to the Blues. i-Perception 9, 6 (Nov. 2018), 204166951880853. https://doi.org/10.1177/2041669518808535

[44] Lee H. Wurm, Gisela Labouvie-Vief, Joanna Aycock, Kristine A. Rebucal, and Heather E. Koch. 2004. Performance in Auditory and Visual Emotional Stroop Tasks: A Comparison of Older and Younger Adults. Psychology and Aging 19, 3 (2004), 523-535. https://doi.org/10.1037/0882-7974.19.3.523

[45] Marcel Zentner, Didier Grandjean, and Klaus R. Scherer. 2008. Emotions Evoked by the Sound of Music: Characterization, Classification, and Measurement. Emotion 8, 4 (2008), 494-521. https://doi.org/10.1037/1528-3542.8.4.494

[46] Zhihong Zeng, M. Pantic, G.I. Roisman, and T.S. Huang. 2009. A Survey of Affect Recognition Methods: Audio, Visual, and Spontaneous Expressions. IEEE Transactions on Pattern Analysis and Machine Intelligence 31, 1 (Jan. 2009), 39-58. https://doi.org/10.1109/TPAMI.2008.52 